\title{
Factor de amenaza y ejes de la percepción de amenaza chilena: ¿Qué política de defensa para Chile?
}

\author{
Cristián Leyton
}

"The territory of a state is the base from which it operates in time of war and the strategic position which it occupies during the temporary armistice called peace.»

Colin S. Gray, 1977.

No obstante los cambios sistémicos globalizantes, los entes estatales siguen inmersos en un virtual estado de naturaleza internacional. Esta característica inmanente a las unidades estatales hace que estos últimos tiendan a percibir cualquier acción, intención y/o capacidad de los Estados que le circundan como potencialmente amenazantes para su seguridad económica, cultural, militar como política. Poco importa el grado de integración y de cooperación interestatal, a partir del momento en que no existe un órgano supraestatal capaz de monopolizar el uso de una fuerza legítima, el factor de amenaza constituirá la característica central en toda relación internacional. Esto significa que el factor de amenaza no puede ser; en ningún caso suprimido, sólo controlado. Por ello, resulta necesario hacer la luz tanto sobre el concepto como sobre los factores de amenaza tradicionales que han afectado la seguridad político-militar del Estado chileno. Antes de ahondar en el concepto de seguridad cooperativa, tan de moda actualmente, primero debemos ser capaces de comprender las fuentes tradicionales que han servido de fundamento, determinado e influenciado el posicionamiento estratégico del país en el concierto de Estados del Cono Sur: Propongo, en conclusión, trabajar el vacuum teórico evidenciado por el campo de los Estudios Estratégicos en Chile. ${ }^{l}$

\footnotetext{
'A principios de la década pasada se avanzó en este sentido. Sin embargo, muy pocos estudios y análisis continuaron la tarea en cuestión cuyo objetivo final era, evidentemente, llenar el vacio en cuanto a las percepcio. nes de amenaza que guiaron, influenciaron y determinaron las políticas militares y estratégicas de los decenios precedentes. Mi trabajo pretende, humildemente, retomar la labor ya comenzada, esta vez desde una perspectiva mucho mas teórica. Consultar, Cruz, Rigoberto y Varas, Augusto (Editores), Percepciones de amenaza y políticas de defensa en América Latina, FLACSO/CEEA, 1993. De manera puntual consultar, Meneses, Emilio., "Percepciones de amenaza militares y agenda para la política de defensa", R. Cruz y A.Varas, FLACSO/CEEA, pp. 365-441.
} 
Minacia, en latín, describe, una palabra, un gesto, un acto a través de los cuales un sujeto expresa la voluntad de hacer daño a otro. ${ }^{2}$ De esta forma, el concepto de amenaza constituye un signo que presagia algún tipo de peligro para la integridad global de una entidad cualquiera. La percepción o percipere, cuanto a ella, aparece como una representación consciente a partir de sensaciones generadas por la expresión de una intencionalidad, esgrimida consciente o inconscientemente por un tercero.

De esta forma el termino de percepción de amenaza tiene un origen sociológico, vale decir; es producto directo de algún tipo de relación humana. En este sentido, la amenaza es un factor constitutivo de las relaciones sociales por cuanto este principio se encontraría, teóricamente, al origen de la formación y emergencia de la vida societal. El estado de naturaleza, avanzado por Thomas Hobbes ${ }^{3}$, comprendido como una situación de guerra de todos en contra de todos en donde el hombre es un lobo para el hombre demuestra el papel jugado por este principio en la constitución de órganos superiores al individualismo humano. La percepción de amenaza a la integridad fisica y moral de los individuos les impulsaría a crear y delegar, conscientemente, en un órgano superior; ciertas libertades que le son inherentes con el fin de que éste les garantice seguridad y paz en sus relaciones interpersonales. Este contrato social Lockiano ${ }^{4}$, garantiza que cualquier conflicto que se produzca al interior de los límites nacionales y soberanos de un Estado serán resueltos por un arbitro-poder judicial-, cuya decisión uiltima deberá ser acatada por los entes en conflicto. Su acatamiento esta asegurado por el monopolio legitimo de la fuerza que detiene el Estado quien es el encargado de resguardar la institucionalidad vigente.

El Estado emerge, entonces, como la solución fiente al permanente estado de inseguridad que caracterizaba la vida pre-estatal. Ahora bien, si a nivel nacional, los individuos están protegidos por el monopolio de la violencia legítima ejercida por el Estado, sobre el plano interestatal, vale decir de

\footnotetext{
2 Diccionario Larousse, versión 1.0, Lrisi Interactive 1997.

3 Thomas Hobbes (Wesport, Wiltshire, 1588 - Hardwick Hall 1679.) Filosofo ingles. Promotor de un materialismo de tipo mecanicista concebía al Hombre con una tendencia natural hacia el temor y el deseo, por ello para vivir en sociedad, este autor consideraba que los Hombres debian renunciar a ciertos derechos en función de un soberano, el cual representaba el orden (Léviathan, 1651).

${ }^{4}$ John Locke (Wrington, Somerset, 1632 - Oates, Essex, 1704).

Otro filosofo ingles. Promotor del liberalismo político, considera que la sociedad reposa sobre un contrato y que por lo tanto el soberano debe obedecer a las leyes que él mismo esta encargado de hacer cumplir (Cartas sobre la tolerancia, 1689).
} 
las relaciones entre unidades estatales soberanas, el factor de amenaza continua, por el contrario, ejerciendo un dominio absoluto, por cuanto, a nivel internacional no existe un ente supranacional detentor del uso de la fuerza legítima. Así como la naturaleza soberana de los Estados les permite dotarse de una organización intema propia, su autonomía frente a las otras unidades estatales les capacita para establecer relaciones de igual a igual. Ambas caracteristicas les otorgan la facultad inica de elegir libremente entre la cooperación o el conflicto con sus semejantes.

En teoría, el linico factor que incidiría en esta elección es la voluntad política de los órganos decisiorios políticos impulsando, a las máximas autoridades del Estado, a adoptar una u otra estrategia, la paz o la guerra, el conflicto o la cooperación.

Inmersos en este virtual estado de naturaleza internacional, los entes estatales tienden a percibir cualquier acción, intención y/o capacidad de los Estados que le circundan como potencialmente amenazantes para su seguriclad, tanto económica, cultural, militar como política. De esta forma, a partir del momento en que no existe un órgano supraestatal capaz, de monopolizar el uso de una fuerza legítima y superior a todos los entes estatales del sistema internacional, el factor de amenaza constituirá la característica central en toda relación internacional.

\section{Introducción al factor de amenaza interestatal}

Pero en términos prácticos, ¿cómo se exterioriza esta percepción de amenaza?

Tal como ha sido expuesto en un comienzo, la amenaza implica intrínsecamente la posibilidad que enfrenta un Estado de perder o verse despojado, ya sea de una posesión material o de una condición -económica, política, o militar- dada. En síntesis, la privación de algo percibido, por las autoridades en cuestión, como gozando de un determinado valor nacional, como el territorio, la restricción o pérdida de soberanía, la suspensión de beneficios económicos, la pérdida de autonomía decisoria y de la independencia política, o bien, la disminución de las capacidades de influencia sobre el plano internacional. 
Tal como podemos observar, el factor de amenaza tiene una doble naturaleza epistemológica. Por un lado expresa y describe una consecuencia (la pérdida de una posesión o condición), pero por otro lado se manifiesta como un medio, vale decir, como un instrumento de poder que será utilizado por un ente estatal para poner en juego la seguridad de su semejante (coerción, disuasión, presión económica, diplomática o política). Si bien, la primacía de un medio de poder por sobre otro dependerá de múltiples factores sistémicos, el que más atrae la atención de los círculos ligados a los Estudios Estratégicos es el instrumento de coerción, vale decir, la utilización efectiva de la fuerza puesto que éste implica el uso de la guerra con el fin de alcanzar objetivos políticos.

\section{El factor de amenaza temporal: real y potencial}

Las fuentes de amenaza percibidas por los círculos decisorios políticomilitares esta determinada por un factor de tipo temporal. Vale decir que, tal como el concepto de ataque sorpresa se subdivide en aquel de tipo preventivo y preemptivo, dependiendo de la inminencia posible de la conflagración, la percepción de peligro para la integridad territorial y soberana de un país se subdivide en una percepción de amenaza real y otra potencial, dependiendo, esta vez, del estado de los preparativos bélicos de un Estado cualquiera.

La amenaza real se refiere, entonces, a un peligro inminente, generalmente militar, medido en días y horas, mientras que la amenaza potencial se refiere, más bien, a cualquier preparativo bélico asociado a políticas declaratorias hostiles poniendo, al mediano y largo plazo, en peligro la seguridad militar económica y/o política- del país. En este caso la amenaza percibida podría materializarse en cuestión de semanas y meses. En resumen, por un lado hablamos de acciones concretas, por otro lado de intenciones.

De esta forma, ¿si la paz es definida por algunos como la ausencia de guerra, la seguridad debe ser comprendida como la ausencia de amenaza? Considero que no. La existencia de un ente supraindividual a nivel nacionalel Estado- no elimina per se el sentimiento de amenaza de los individuos, sino que más bien otorga un cierto grado de seguridad, según el cual, cualquier conflicto que se genere podrá ser resuelto por un tercer ente, en este caso, por los órganos judiciales. En el caso de no ser acatado el fallo, no es el demandante quien se hará cargo de hacerlo cumplir, sino que una entidad que les es superior a ambos. De esta forma, el individuo delega el uso de la fuerza 
coercitiva al Estado, asegurando de esta forma un clima propicio para la prosperidad socio-economica y el desarrollo del conjunto de sus potencialidades.

En este sentido, la seguridad no debe ser comprendida como la ausencia de amenazas militares y no militares, sino que más bien como la ausencia de fuentes de amenaza reales. En otras palabras, el factor de amenaza siendo una característica inherente del sistema internacional sólo puede ser disminuido, pero en ningún caso suprimido. Por ello, un Estado goza de una seguridad relativa en el momento en que los entes decisorios no perciben un peligro inminente para su soberanía, su sobrevivencia y/o sus instituciones. La amenaza existe pero es potencial, en el sentido en que podría en un futuro lejano desarrollarse y tomar forma, pero que, dadas las condiciones actuales, no merita la toma de medidas de protección territorial y soberana extraordinarias. ${ }^{5}$

Una característica para retener: tanto la amenaza potencial como la real no son inclusivas en el sentido que no identifican a ningún actor específico como fuente de amenaza. Todos los actores del sistema internacional, estatales o no, pueden eventualmente evolucionar hacia una amenaza a los intereses nacionales.

El factor de hostilidad, ocupa, en este sentido, un papel central. ${ }^{6}$ Es este factor de animosidad el que introduce la diferencia entre la percepción de seguridad -amenaza potencial-y de inseguridad -amenaza real. ¿Que hace que Canadá no se sienta amenazado militarmente por los Estado Unidos, gran superpotencia militar mundial? ¿Cómo se explica que Bélgica no se sienta amenazada militarmente pese a su delicado emplazamiento geo-estratégico, en medio de dos colosos militares, tales como la Francia y el Reino Unido? En cambio, ¿ Que hace que el Estado Estoniano si se sienta amenazado por el Estado Federal de Rusia, pero no así el Bieloruso?

La respuesta nos es entregada por el factor de hostilidad interestatal. Existe inseguridad en el momento en que algún grado de hostilidad se establece entre dos o más Estados. ¿Pero sólo eso basta? Raramente la hostilidad entre Estados soberanos se limita sólo a un antagonismo estático. En efecto, la

\footnotetext{
s En resumen, podríamos decir que el factor de amenaza es por naturaleza estructural y orgánico, forma parte de los mecanismos y de la estructura misma del sistema internacional. Una visión mas detallada de este fenómeno nos es presentada por Klaus Knorr, "Threat Perception", Historical Oimensions of National Security Problems, The University Press of Kansas, 1987, 78-117.

6 Jean Barrea nos entrega una descripción epistemológica de gran valor en su obra, Theorie des Relations Internationales, 3 era Edición, Artel, 1994, paginas 126-146. Ver además, J. Garnett (Edit.), Theories of Peace and Security, A Reader in Contemporary Strategic Thought, Londres, Macmillan, 1970, 378 paginas.
} 
mayor parte del tiempo este factor se asocia a la adquisición de ciertas capacidades bélicas, y se potencia, a la vez, a través de determinadas políticas declaratorias dejando presagiar algún tipo de intención en usarlas.

En este sentido, es necesario establecer que tanto las intenciones como las capacidades de las unidades estatales son por naturaleza irregulares, vale decir, que éstas tienen la característica de cambiar abruptamente en el tiempo. Esta aseveración tiene aún más significado entre Estados habiendo mantenido, en un pasado cercano, algún tipo de relación conflictual. Tal es el caso alemán, por ejemplo. La reunificación alemana simbolizó, no solo, el fin del glacis ${ }^{7}$ soviético en Europa del Este, sino que también, el resurgimiento de una Alemania elevada al rango de gran potencia económica y militar, europea y mundial. Este último hecho provocó la reacción de las autoridades francesas, temerosas de ver este nuevo coloso, una vez más, unificado y libre en el corazón mismo de Europa. Si los órganos estatales franceses no lograron ejercer un control sobre esta unificación al intentar negociar la reunificación si lograron, al menos, mantener a Alemania contenida dentro de una organización militar, la OTAN. ${ }^{8}$ Ahora bien, no sólo una percepción de amenaza militar impulsaba el ballet diplomático que mantuvo el Quai D'Orsay durante los primeros días y meses del proceso de reunificación germana, sino que también una amenaza de carácter político: Francia perdía su rol protagónico en los asuntos europeos, dejaba de ser el pivote político del conjunto de Europa Occidental.

La historia no ha dado razón a la percepción de amenaza francesa: Alemania cambió su potencial pero no sus intenciones. Diferente fue el caso Alemán durante el Tercer Reich. Hitler no cambió, declaratoriamente, sus intenciones pseudopacifistas (aunque lo hizo en secreto), pero sí, y en forma reservada, sus capacidades. El resultado de esta transformación de la amenaza, de una potencial a una real, comenzó a verse desde 1939 en adelante. En este sentido, la identificación de las intenciones y la evaluación de las capacidades del adversario potencial son fuente de atención de la mayor parte de los

\footnotetext{
7 Por glacis debemos entender un espacio o zona geográfica emplazada al exterior de las fronteras políticas nacionales ubicándose entre entes estatales hostiles entre si y utilizada, por consiguiente, a fines de seguridad militar. En relación a este tốpico consultar, Débié, Frank.,. “À quoi ça sert la Géostratégie?", Stratégique, F.E.D.N, vol. 2, no.90, Paris, 1990, paginas 85.88 .

${ }^{8}$ No obstante el hecho que las mismas autoridades alemanas dejaron ver, desde los primeros meses del proceso de reunificación, la intención de manterierse dentro de la Alianza Atlántica.
} 
órganos estatales por cuanto, éstas, permiten al Estado prepararse política, diplomática y militarmente ante cualquier eventualidad.

Ahora bien, no obstante el hecho que las capacidades bélicas de un Estado pueden permitir a otro conocer los alcances y límites de la amenaza a afrontar (una diferencia existe entre un Estado que adquiere mísiles balísticos de largo o mediano alcance tierra-tierrra y otro que adquiere sistemas de mísiles tierra-aire, ${ }^{9}$ el primero es por naturaleza ofensivo, el segundo de defensa anti-aérea), el conocimiento de las intenciones políticas y militares de un ente estatal es una tarea mucho más subjetiva. En efecto, el proceso que conlleva a descifrar si éstas son hostiles o no está sujeto a un alto grado de subjetividad por cuanto es tarea de la inteligencia nacional determinar el tipo de amenaza pudiendo enfrentar un ente estatal, y ésto, a corto, mediano y largo plazo. Es esta Inteligencia la que debe, entre los ruidos, aislar las señales y así descifrar las verdaderas intenciones vecinales. Si los medios actuales de adquisición de la información dependen, en su mayoría de medios electrónicos, el procesamiento sigue en manos de seres humanos con prejuicios, sumidos a la influencia ideológica, así como a pensamientos deseados (conocido como wishful thinking). Breve, imperfecta, difícilmente alcanzable, pero absolutamente necesaria en un sistema internacional marcado por el estallido de la amenaza y la difusión de la potencia..

Este antecedente nos conlleva a identificar otra característica del factor de amenaza.

\section{El factor de amenaza general y específico}

Así como existe una disuasión general (General Deterrence), vale decir, una política de Estado tendiente a declarar públicamente que es una política de defensa disuasiva la que se adoptará ante cualquier fuente de amenaza, y una disuasión específica (Specific Deterrence), que describe e identifica las acciones precisas pudiendo ser percibidas como fuente real de amenaza y por

\footnotetext{
9 No obstante el hecho que al adquirir dichos sistemas de defensa antiaérea, el Estado se proponga utilizarlos como paraguas protectores en una estrategia de penetración en territorio adversario, tal como sucedió en la guerra de Yom Kippur en 1973. En esta ocasiôn las fuerzas egipcias adquirieron sistemas avanzados Sam-6 soviéticos con el fin de proteger el avance de sus tropas en el momento de atravesar la rivera occidental del Canal de Suez ocupado por los israelies. No obstante esta excepción, mas alarma causa aún la adquisición de algún vector con características de ataque estratégico que aquel destinado al uso táctico, y ello, a excepción de países que no poseen profundidad estratégica.
} 
ende, pudiendo exigir el uso de la fuerza por el Estado amenazado, existe también una amenaza general y otra especifica.

Por amenaza general debemos entender la identificación de una fuente de amenaza potencial. En otras palabras, el Estado prioriza y jerarquiza a los Estados pudiendo constituir una amenaza para sus instituciones. Ello dependiendo de diversos factores, tales como el pasado histórico, el choque actual y/o tradicional de intereses, intenciones percibidas como expansionistas, la creación de zonas de influencia, la adquisición de glacis militares ofensivos, etc.

En cambio, hablamos de amenaza específica, a cualquier acción percibida como hostil que emane de un ente estatal con quien existe un factor de hostilidad en vigor, pudiendo, a corto plazo o en la inminencia, convertirse en un peligro para la integridad territorial o soberana del país. Así, cuando la amenaza general evoluciona por medio de acciones y de políticas declaratorias hostiles acompañadas, en la mayor parte de los casos, por la adquisición de nuevo material de guerra, ésta se convierte en una de tipo especifica. En este caso nos referimos a una concentración inhabitual de tropas vecinales en zonas fronterizas, a su movimiento y despliegue, al bloqueo de ciertos puntos geoestratégicos sensibles o vitales para la estructura económica local, la firma de alianzas militares a carácter ofensivas, la adquisición de capacidades de ataque estratégicos y ofensivos, ya sean éstas balísticas o aviónicas, etc.

En pocas palabras, nos referimos a una acción actual que describa algún tipo de riesgo inminente para la seguridad nacional.

De esta forma, la amenaza general describe la fuente tradicional de amenaza en un estado pasivo (hasta que el factor de hostilidad disminuya o desaparezca convirtiéndose en una amenaza potencial), mientras que la amenaza específica se refiere a un tipo de amenaza en estado activo, operacional. Por un lado hablamos de intenciones tradicionales, por así decir, mientras que por otro lado de acciones concretas.

Ahora bien, si la amenaza está subdividida por factores de tipo temporal, también lo está por uno de orden cualitativo.

\section{El factor de amenaza limitado y absoluto}

En efecto, la percepción de amenaza estatal esta ligada al grado de peligro a enfrentar por la unidad estatal. Esta puede ser limitada o absoluta.

Por limitada entendemos la obtención de beneficios económicos, políti- 
cos o militares destinados, ya sea, al ejercicio de algún tipo de influencia a nivel vecinal, regional o mundial, a restablecer o reforzar el estatus quo sistémico, a modificar el equilibrio de poder -militar o de intereses-, entre otros. En términos prácticos, y a título de ejemplo, podemos decir que la mayor parte de los conflictos en el sistema internacional obedecen a esta clasificación.

Cuando hablamos de percepción de amenaza absoluta hacemos referencia a la eventualidad de, no sólo, ver limitada la soberanía nacional sobre determinadas competencias estatales (pérdida de poderío defensivo u ofensivo), o sobre específicas zonas geográficas, sino que verse despojado de todo poder soberano, vale decir, la desaparición como Estado, y por ende, todas las consecuencias que de este hecho se desprenden.

Este fenómeno puede ser generado por dos factores:

- Por un lado, como producto de una invasión, conquista y ocupación de ciertas zonas vitales, tales como la capital política de un país, por ejemplo -la desmembración constituyendo su más extrema expresión.

- Por otro lado, como producto de la internupción de la continuidad territorial de un país.

En general, cuando nos referimos a la amenaza absoluta hacemos referencia, a tres casos específicos:

- A la amputación de ciertas zonas geográficas esenciales para la supervivencia del ente estatal, especialmente, de zonas geoeconómicas vitales para el funcionamiento de la estructura económica nacional,

- A la pérdida de zonas geoestratégicas fundamentales para la defensa territorial de un centro vital,

- A la adquisición, por un Estado percibido como hostil, de zonas geopolíticas vitales pudiendo otorgarle una capacidad de proyección de potencia mayor y de esta forma inhibiendo la propia..

A título de ejemplo, podemos citar el caso de algunos países monoexportadores y oligoexportadores como Arabia Saudita. En este caso, no es necesario ocupar Ryahd, su capital, para amenazar su existencia como Estado soberano, la sola ocupación de sus zonas petroleras concentradas en su mayoría al Este del territorio nacional constituiría la vía para tal efecto al despojarla de cerca de $80 \%$ de su PIB en una sola acción de fuerza

El factor de amenaza constituye, tal como lo hemos visto, la principal consecuencia del estado de inseguridad en el cual se bañan las relaciones interestatales. Frente a esta realidad, el Estado reacciona adoptando una serie de 
medidas a carácter político, económico, militar e incluso cultural, dependiendo de la coyuntura internacional, de las capacidades nacionales, de la voluntad política estatal así como del grado de autonomía política del ente estatal.

La respuesta político-militar frente al factor de amenaza se expresa, generalmente por la vía de la llamada política de defensa, la cual encierra, en si, una amplia gama de subrespuestas, las cuales están asociadas a una gran política estatal. Entre las más adoptadas podemos citar, el rearme, la disuasión, la formación de alianzas ofensivas o defensivas, la modernización militar, el desarme (por la fuerza, vale decir la guerra, o la negociación), el control de armamentos, la conciliación, o bien, la acomodación.

Es así como, el Estado, frente a una amenaza militar, real o potencial, de naturaleza exógena formula, por medio de la Política de Defensa, un conjunto de medidas a carácter defensivo con el fin de neutralizar las fuentes de peligro para la integridad territorial del país y de su soberanía.

En este sentido, es necesario establecer que toda política de defensa está influenciada y/o determinada por la relación que se establece entre tres factores constitutivos de la teoría de seguridad: la configuración territorial, el factor de hostilidad interestatal, y el equilibrio militar regional. ${ }^{10}$ El valor estratégico acordado a cada factor, en la formulación de la doctrina militar (vale decir, los principios que guían el uso de la fuerza militar), depende, generalmente, de dos fenómenos:

- Del grado de hostilidad interestatal regional o vecinal percibido por cada unidad estatal. La importancia de los tres factores antes mencionados radica en el hecho que, en conjunto, generan una síntesis, la cual es central para comprender las relaciones de competición y/o de cooperación vecinal. Esta síntesis se encuentra materializada en la percepción de amenaza generada por los órganos decisorios político-militares estatales. Es esta percepción de peligro la que influencia y/o determina que, dadas ciertas condiciones, la Ocasión de servirse de una fuerza coercitiva emerja como la solución última

\footnotetext{
${ }^{10}$ En ciertos casos, el equilibrio de intereses juega un rol central en la percepción de amenaza así como en la formulación de una política de defensa. Tal fue el caso de la guerra de Yom Kippur o de Ramadan en 1973, entre israelíes y fuerzas aliadas siro-egipcias. El Estado Egipto consciente que el equilibrio militar favorecila al Tshal israelí decide, de igual forma, atacar el Estado Hebreo. La racionalidad de este acto se explica por el desequilibrio existente a nivel de intereses: para los egipcios el status quo de 1967 era lejos más intolerable que una derrota militar en 1973. Ello gatilla su implicancia en una nueva guerra con Israel, en donde la victoria política del estado Egipcio se impuso por sobre la militar Israelita al obligar, a este ultimo, a devolverle el Sinaí gracias a la intervención de toda la comunidad internacional en la resoluciōn de la problemática heredada de la guerra de los Seis Días en junio 1967.
} 
en un conflicto de intereses que, hasta ese entonces, sólo estaba limitado a una fricción eminentemente política, económica o diplomática.

En este sentido son las posibles intenciones secundadas por ciertas capacidades las que determinan el valor asignado a cada uno de los factores antes señalados.

- El valor estratégico también depende de las capacidades materiales y no materiales nacionales. Por capacidades materiales debemos entender el conjunto de los recursos económicos de un país, el capital demográfico o humano del mismo, las capacidades bélicas letales o de transporte y de movilización de sus fuerzas armadas y de orden, entre otros medios.

Por el contrario, hablamos de medios no-materiales al grado de unidad nacional reflejado por un país cualquiera, a la capacitación de los individuos formando parte los órganos estatales de decisión política, el grado de culturización de la población nacional, la capacidad de autonomía política del Estado vis-à-vis de la comunidad de potencias mundiales, el grado de unidad nacional en tiempo de crisis, la eficacia diplomática del Estado, etc.

Tal como podemos apreciar, no todos los factores de seguridad tienen el mismo valor en la formulación y elaboración de políticas de defensa. Así como en determinados casos, son el conjunto de factores los que influyen y determinan la política de defensa de un país, tal es el caso de Israel por ejemplo, en otros sólo determinados factores dictan la implementación de ciertas políticas militares, tal como fue el caso de la confrontación indirecta sovietoestadounidense durante la Guerra fría.

En el primer caso, la configuración territorial del Estado hebreo sumada al grado de hostilidad interestatal impulsa el mantenimiento por Israel de un equilibrio militar regional fundado en un desequilibrio estratégico en su favor. Los factores en cuestión dictaban, al Estado israelí, una política de defensa doctrinalmente defensiva, pero operacionalmente ofensiva fundada sobre un sistema de casus belli de naturaleza estratégica así como la creación de glacis militares en todas sus fronteras políticas internacionales. En el caso soviético-estadounidense, el factor determinante, en cambio, era el equilibrio militar fundado, esta vez, en un balance nuclear cualitativo, pero en un desequilibrio militar a nivel de las fuerzas convencionales, desequilibrio compensado por una supremacía cuantitativa soviética pero con una superioridad cualitativa occidental.

Tal como lo hemos visto, la configuración territorial de un país, tal como su emplazamiento y su configuración física, no es sinónimo de amenaza si 
ésta no está asociada al factor de hostilidad interestatal. Si este factor existe entre unidades estatales distantes geográficamente pero sin medios letales de expedición, intercontinental o de largo alcance, la amenaza puede, eventualmente, permanecer a nivel de amenaza potencial hasta el momento en que el Estado A se dote de una capacidad balística o aviónica de largo alcance. Ahora bien, si la hostilidad se establece entre Estados vecinales, la amenaza pasa a ser explícita, real e inmediata, tal es el ejemplo indo-pakistaní. Si agregamos a este escenario una configuración geoestratégica tal que los centros vitales nacionales del Estado A son completamente vulnerables como consecuencia de una falta de profundidad estratégica horizontal, la percepción de amenaza se convierte en absoluta. En este caso, dos macroestrategias y dos microestrategias pueden ser adoptadas por el Estado A:

- Sobre el plano de las macroestrategias, hablamos de la puesta en obra, ya sea, de un sistema de disuasión -conflicto indirecto y controlado- o de un régimen de cooperación -minimal o avanzado:

En cuanto a las microestrategias, y no menos importantes:

- Por un lado, la operacionalización de una política de defensa activa, de prevención y de anticipación.

- Por otro lado, la adopción de una política de defensa pasiva, de absorción y de contraataque.

La primera política tiene como objetivo desarmar la fuente de amenaza antes que ésta haga uso de su potencial militar, mientras que la segunda se da como objetivo último neutralizarla por medio del intercambio de territorio por tiempo esperando, idealmente que en un mediano plazo, las condiciones para la eventual desaparición o disminución del factor de hostilidad que les enemista se instale, desarrolle y solidifique.

En este sentido, $¿$ cual ha sido la influencia de los factores geopolíticos y geoestratégicos en la formulación de la política de defensa chilena durante el período 1973-1990? ¿En qué medida la percepción real o potencial de una amenaza para la integridad física y soberana del país era determinada y/o influenciada por factores geográficos? En resumen, ¿en qué medida la geopolítica así como la geoestrategia nacional determinaran Qué política de defensa para Chile?

Si bien es cierto, la tarea de responder a esas preguntas sugiere un análisis detallado del tema, interesante sería, al menos, resaltar en esta ocasión las principales fuentes de amenaza que alimentan la política de defensa chilena 
en el período en cuestión. En este sentido, y para tal afecto, sería preciso bosquejar las potenciales y reales fuentes de amenaza para Chile. ¿Cuales y cómo se asimilan las amenazas percibidas por las autoridades chilenas desde el punto de vista geoestratégico?.

\section{Geoestrategia territorial chilena y percepción de amenaza}

"Chile una larga y angosta faja de tierra", esta ya tradicional frase resume a la perfección las dos principales características geográficas del territorio chileno. Con una frontera de $6.280 \mathrm{kms}$ de largo, aproximadamente, y una extensión de $756.626 \mathrm{kms}$, el ancho máximo no sobrepasa en ningún punto del territorio nacional los $435 \mathrm{kms}$, siendo su estrechura promedio los $180 \mathrm{kms}$.

Estos datos no tienen ningún valor político ni militar si no se les asocia a dos factores: Por un lado, al factor de amenaza interestatal vecinal, y por otro lado, a un factor geoestratégico, en este caso, al emplazamiento de los nilcleos o centros vitales del país.

A continuación, analizaremos los principales antecedentes geopolíticos nacionales, así como los factores geoestratégicos que determinan e influencian la percepción de amenaza estatal chilena. Luego será cuestión de abordar, someramente, la respuesta político-estratégica que, en principio, debería guiar la formulación de una política de defensa.

\section{Principales factores geoestratégicos del territorio chileno}

Digamos que Chile goza de una profundidad estratégica vertical, pero carece de toda profundidad estratégica horizontal. ${ }^{\text {}}$ Por profundidad estratégica debemos entender, la distancia que separa los núcleos vitales ${ }^{12}$ estatales de las fronteras políticas internacionales. ${ }^{13} \mathrm{Si}$ bien es cierto, esta definición nos permite comprender el sentido amplio del término en cuestión, sería necesario agregar un último elemento: la configuración geográfica de la fron-

\footnotetext{
"Evidentemente, esta aseveración es relativa, puesto que depende exclusivamente del factor de hostilidad interestatal. No obstante esta remarca, la asumiremos como totalmente valida a fines del presente análisis. 12 Por núcleos vitales, debemos entender, los centros de concentración de las principales agrupaciones demográficas, económicas, políticas y militares de una naciön.

${ }^{23}$ Definición inspirada de Aharon, Yariv., "Strategic Depth", The Jeusalem Quarterly, no. 17, otoño 1980, paginas $23-31$.
} 


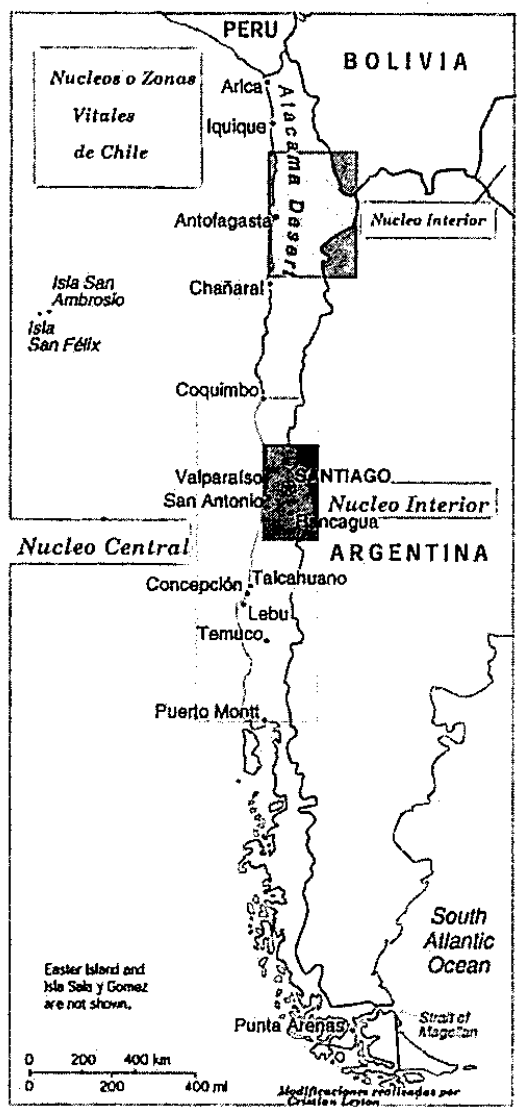

tera intemacional. ${ }^{14}$ No sólo la extensión que separa las principales ciudades de un país puede determinar o influenciar la percepción de amenaza, sino que también la configuración morfológica de la frontera, pudiendo, ésta, gatillar una aventura belicista o bien neutralizarla. En este caso, y a título de ejemplo, las fronteras políticas internacionales del Estado Confederado de Suiza tienen un decisivo efecto disuasivo al favorecer una defensa en la profundidad topográfica montañosa helvética -sumada, evidentemente, a las modernas capacidades bélicas suizas así como a su sistema de conscripción permanente soldado-ciudadano. De esta forma, la posibilidad de poner en práctica una estrategia de atrición frente a un enemigo externo parece haber disuadido, hasta el momento, cualquier intento de invasión.

Tal como ya ha sido mencionado, la existencia y el emplazamiento geográfico de los núcleos vitales de los Estados determinan o influencian la percepción de amenaza.

En el caso chileno, dos núcleos vitales pueden ser identificados:

- La zona cuprífera emplazada en el extremo norte del país,

- La zona central.

El territorio nacional goza de una profundidad estratégica Norte-Sur, pero carece de toda profundidad estratégica en su eje Este-Oeste. En este sentido cabe señalar que en la zona central, conocida como el Valle Central, la distancia entre la zona costera y la frontera internacional varia sólo entre 40 y $80 \mathrm{kms}$ de ancho.

14 La problemática ligada a la influencia que ejerce la configuración morfológica de la frontera en la seguridad militar estatal será tratada ulteriormente en un paper a aparecer próximamente. 
Ahora bien, la profundidad estratégica vertical del territorio chileno es relativa por cuanto sólo lo es en relación a un núcleo vital, vale decir, su capital y no en su centro vital económico, la segunda región de Antofagasta. Analicémoslo.

- El núcleo vital de la zona norte: La zona minera de Chile se concentra entre las 1era y 3era regiones, siendo la más importante y principal, la Región de Antofagasta, la cual concentra el 100\% de la producción de cobre del país. Su importancia radica en la magnitud actual de las operaciones de extracción, en la diversidad de la producción, así como en el nivel histórico de las inversiones que alli se han realizado.

¿Que razón conlleva a considerar, esta zona, como un núcleo vital?

Para responder tenemos que tomar en cuenta el papel jugado por esta zona en la estructura económica chilena, vale decir los allí recursos generados y su importancia para la economía nacional.

Establezcamos que cerca de $42,4 \%$ del total de las exportaciones nacionales es generado por los ingresos provenientes de la venta del cobre producidos en esta zona, y ello, sin olvidar que otro $8 \%$ es generado por la explotación de otros minerales (Nitrato y hierro, principalmente) procedentes de esta misma región ${ }^{15}$. Esta situación crea, como es lógico, una alta dependencia de la economía nacional hacia los recursos que allí se generan, por cuanto aproximadamente un $60 \%$ del PIB es producido en estas tres regiones del norte chileno.

De esta forma, dada la estructura oligoproductora de la economía chilena, ${ }^{16}$ la Región Cuprífera constituye un núcleo vital por cuanto su amputación, temporaria o permanente, del territorio chileno podría, no sólo, paralizar la estructura económica del país, sino que destruir las bases del conjunto de su economía nacional. Este fenómeno implicaría diferentes consecuencias para el poder nacional: Despojar de toda capacidad adquisitiva logística -en

is Segün la Comisión Chilena del Cobre y el World Metal Statistics la producción chilena de cobre fue, en 1998 , de $3.686,9$ toneladas métricas. En cuanto a la inversión extranjera focalizada hacia el sector minero, el Comité de lnversiones Extranjeras estima que durante 1997 la inversiốn autorizada fue del orden de los 7.893. 7 millones de dólares, mientras que la inversión efectiva alcanzó los 1.705.5 millones de dólares. Para información mas detallada visitar el sitio web de Cochilco, www.cochilco.cl, Al $42,4 \%$ habria que agregar el $20,6 \%$ asociado al sector agropecuario, el $12,6 \%$ al sector forestal, el $10,9 \%$ al sector pesquero,y el $13,2 \%$ al industrial. Banco Central de Chile sobre la base de los Informes de Aduana (Embarques), 1999. Dirección General de Relaciones Económicas Internacionales, Dirección de Estudios (Noviembre, 1999).

${ }^{16} \mathrm{Y}$, señalemos, que hasta recientemente monoproductora en el sentido en que un solo producto ha representado históricamente más del $50 \%$ del calculo del PIB en la estructura económica nacional: del guano, pasando por el salitre y terminando con el cobre. 
caso de conflagración general-, diezmar gravemente la capacidad de negociación política de posguerra de los órganos estatales chilenos, menoscabar la capacidad de resistencia de la población civil así como generar un clima de crisis social interna sin precedente en la historia chilena pudiendo obligar al Estado chileno a ceder frente a demandas exteriores intolerables.

En resumen, su ocupación o en caso contrario, su disgregación -temporal- del Estado chileno por una potencia extranjera significa de facto el fin de la soberanía nacional por sobre el heartland ${ }^{17}$ mismo de toda la estructura que sostiene la economía chilena.

- El núcleo vital de la zona central: La existencia de vacuums demográficos en los extremos del territorio chileno así como una concentración demográfica y político-administrativa orientada hacia el centro del país hace de la zona central un núcleo vital imprescindible para la supervivencia del Estado chileno como ente soberano.

Dos factores alimentan este estatus particular:

- Es, en esta zona central, que el heartland coincide con la capital nacional político-administrativa del Estado chileno. No solo la sede del gobierno se encuentra emplazada en esta zona, sino que además las sedes de todos los Estados-mayores de las Fuerzas Armadas, así como los de Orden y Seguridad interior.

Cabe señalar, además, lo desventajoso de la distribución geoestratégica de los centros decisorios estatales tales como el Palacio de Gobierno así como los edificios de las FFAA. En efecto, la mayor parte de estos se concentran en radio no mayor a los 500 metros por lo que su ubicación sigue la tendencia a la concentración de los centros vitales nacionales en espacios reducidos y evitando con ello la dispersión.

- Sobre el plano demográfico esta zona central se subdivide en dos grandes sectores: Por un lado encontramos lo que podemos llamar el núcleo central, y por otro, el núcleo interior.

- El núcleo central comprende desde la IV Región de Coquimbo hasta la X Región de Los Lagos. Es entre estas regiones que la mayor parte de la po-

\footnotetext{
" Harold Mackinder introdujo el concepto de Heartland o zona pivote con el fin de definir la principal zona geográfica de concentración del poder político, económico y militar de una nación. Mackinder, Halford, Democratics Ideals and Reality: A Study of the Politics of Reconstruction, Londres, Constable, 268 paginas. Consultar además, Claval, Paul., Géopolitique et géostratégie, Paris, Édition Nathan, 1994, 338 p.
} 
blación chilena está emplazada. ${ }^{18} \mathrm{El}$ peso demográfico de esta zona es tal que aproximadamente 9 millones de personas sobre un total de 14 viven al interior de estos límites regionales. Este gran núcleo central concentra, además, las más importantes urbes demográficas del país a lo que se le asocia, naturalmente, los principales centros económicos, financieros y comerciales. ${ }^{19}$

- Cuando hablamos de núcleo interior nos referimos a dos regiones bien precisas, la Región Metropolitana y Valparaíso. Estas dos regiones concentran más del 50\% de toda la población del país. En este sentido, cabe señalar que Santiago concentra el $37 \%$ de la población de toda la nación, así como $45 \%$ de toda su población urbana. En cuanto a la V Región de Valparaíso, ésta concentra más de 1 millón trescientas mil personas, siendo la segunda región más poblada del país.

De esta forma, la ocupación del núcleo interior decapitaría, en términos estratégicos, al organismo estatal nacional. En cuanto al núcleo central, cualquier acción bélica que le sea dirigida podría menoscabar decisivamente la capacidad de resistencia de la población por los efectos psicológicos sobre la moral del esfuerzo de guerra civil.

En términos generales, y tal como podemos apreciar, Chile es por naturaleza bicéfalo, con dos centros vitales: tanto la fuente principal de sus capacidades como sus centros de decisiones político-estratégicas se concentran en dos espacios extremadamente limitados en cuanto a superficie, pero extralimitados en cuanto a la extensión de las líneas fronterizas a proteger. Estos antecedentes son agravados por la proximidad de los centros vitales en cuestión a de las fronteras políticas vecinales. En el caso específico de la capital nacional, la profundidad estratégica en su eje oeste-este no existe por cuanto su límite administrativo coincide con los límites internacionales del país.

Ahora bien, si analizamos los núcleos interiores de la zona cuprífera del norte chileno veremos que la distancia entre los principales yacimientos de cobre, y los puntos de transporte así como de redes viales que les están asociados en ningún punto sobrepasan los $50 \mathrm{Km}$. De esta forma, las distancia en

\footnotetext{
18 Según el censo de 1992 la población total era de 13.321 .803 millones de personas, las estimaciones para 1995 eran de 14.237.000 millones, de estas, se estima que un $90 \%$ viven en una franja territorial de tan solo $1000 \mathrm{kms}$ de largo por aproximadamente $180 \mathrm{kms}$ de ancho (núcleo central).

'9 Entre las ciudades más importantes encontramos Santiago, la Capital nacional con cerca de 4. 295.593 millones de personas, la cjudad de Concepción, importante centro agrícola e industrial del país con 326 , 789 personas, Viña del Mar con 303,589 personas, y Valparaiso, principal centro portuario del país con 274,228 personas aproximadamente.
} 
su eje este-oeste se limita a unos cuantos kilómetros, mientras que en su eje norte no más de $200 \mathrm{Km}$. En ambos casos, la profundidad estratégica no permite una defensa en profundidad sin sacrificar, temporariamente, los nícleos vitales interiores del Estado chileno, lo que es, por esencia, prohibitivo.

Pasemos de esta constatación geoestratégica a los principales ejes de la percepción de amenaza chilena.

\section{Principales ejes de la percepción de amenaza chilena}

La tarea que implica analizar los principales ejes de la percepción de amenaza chilena no puede hacerse en un vacío histórico ni coyuntural, sin embargo, a fines de análisis, nos limitaremos a exponer los principales ejes teóricos de la percepción de amenaza nacional, sin apoyarnos, esta vez, en un estudio de caso.

Así como las relaciones comerciales de un país evolucionan a lo largo del tiempo, así lo hace la percepción de amenaza. Sin embargo, así como un país puede gozar en un momento dado y durante un período histórico, en particular, de una bonanza económica, ello no cambia, necesariamente, los fundamentos de la estructura económica nacional. Un Estado cuyas estructuras económicas son, por esencia, oligo y monoproductoras estará siempre expuesto a una mayor vulnerabilidad política exógena así como a una mayor fragilidad socio-política interna, y ello, no obstante una prosperidad económica coyuntural.

De esta misma forma, el factor de hostilidad, cuando existe, puede expresarse en forma potencial, pero también en forma real, inminente. En otras palabras, la amenaza no deja de existir por el solo hecho que ésta no se expresa en la acción, intención o capacidad, sino que existe por el solo hecho que las relaciones internacionales están inmersas en un sistema anárquico en donde el uso de la violencia constituye, aún hoy en día, el último recurso de resolución de conflictos.

En este análisis general, será cuestión de resaltar los principales ejes de la percepción de amenaza chilena. No se trata, en ningún caso de retrasar la evolución de la percepción de amenaza nacional, sino que más bien hacer resaltar las variables constantes que dicen relación con los factores de amenaza a la integridad territorial y soberana chilena, ello siempre desde un punto de vista vecinal y no extra-regional. 


\section{Chile y el factor de amenaza vecinal}

Digamos en un primer momento que la percepción de amenaza chilena es dicotómica: Esta, en teoría, se expresaría en términos de amenaza limitada, pero en términos prácticos, toma, más bien, la forma de amenaza absoluta.

En efecto, la percepción de amenaza es limitada por cuanto lo que estaría en juego es la amputación de los dos vacuums demográficos del país. Por un lado, el extremo sur del territorio nacional, por otro, su extremidad norte. Los nicleos vitales interiores no estarían amenazados directamente, en lo que se refiere a una posible invasión, conquista y ocupación, sino que más bien las zonas débilmente pobladas en relación con los núcleos centrales.

$\mathrm{La}$ adquisición por Chile de las regiones norteñas ricas en minerales a comienzos de siglo habría creado en los círculos políticos y militares chilenos la necesidad de instaurar una política vecinal de estatus quo, ${ }^{20}$ mientras que estos mismos círculos nacionales percibirían a los Estados vecinos norteños como unidades estatales revisionistas del orden territorial de la posguerra del Pacífico. No sólo las políticas declaratorias vecinales justificarían esta percepción de amenaza, sino que también sus capacidades bélicas en determinados períodos históricos recientes. Es así como en la zona del extremo sur, no sólo las políticas declaratorias y las capacidades bélicas transandinas han incidido en determinados períodos históricos en esta percepción de amputación territorial, sino que también la voluntad política de transformar la amenaza en hechos concretos. En este senticlo, sólo el factor de Oportunidad habría impedido que tal amenaza se materialice en distintos períodos históricos recientes. En este sentido, un factor de amenaza clave pudiendo alimentar esta percepción de amputación territorial es el cébil poblamiento de ambos extremos del país. En ambas zonas, la absorción demográfica, en caso de ocupación, no constituiría un problema humanitario insoluble para los países vecinos, contrariamente a los casos de Kosovo o de Bosnia Herzegovina, por ejemplo.

¿A Ahora bien, en que medida esta amenaza no sería, en términos prácticos limitada sino que más bien absoluta? Tal como ya ha sido expuesto, el peligro de amputación territorial no recaería en el centro del país, más precisamente, en su nuicleo vital interior, sino que más bien en la periferia del extre-

20 En otras palabras, ni la Política Exterior ni la de Defensa se dan como objetivo ültimo la adquisición de extensiones territoriales adicionales, en este sentido el Estado chileno declara no tener ninguna pretensión territorial. 
mo norte y sur del país. No es la capital político-administrativa del Estado que estaría en juego, sino que más bien zonas que le están muy distantes. Si esta situación se diera en un Estado que cuenta con una profundidad estratégica multidireccional, como Rusia, por ejemplo, la ocupación de sus espacios periféricos no pondría en peligro la existencia del Estado como ente soberano. Pero la situación geoestratégica chilena dista mucho de la rusa. La ocupación o amputación del extremo norte chileno significa, tal como ya lo hemos planteado, el desmoronamiento de toda la estructura económica chilena como consecuencia del carácter bicéfalo del poder nacional. Si tomamos como referencia, esta vez, el extremo sur, la pérdida de esta zona significaría, en términos prácticos, perder no sólo el acceso a la Antártica chilena, sus aguas ricas en recursos-y los pasos hacia el Atlántico, sino que ésto abriría a la armada vecinal la puerta de acceso a nuestras costas en toda su extensión y con todas las consecuencias que se desprenden.

Estas razones explican el por qué de la amenaza absoluta: la existencia del Estado chileno mismo como ente soberano estaría en juego.

Otra característica propia al medioambiente geoestratégico chileno es la posibilidad real de ver, no solo ocupada sus zonas vitales, sino que también de ver interrumpida su continuidad territorial. En efecto, la angostura y extensión vertical del territorio chileno posibilitan la ocupación de franjas territoriales pudiendo de esta forma, literalmente, cortar al país en dos. Tal como ya ha sido mencionado anteriormente, en la zona central la extensión este-oeste varia tan solo entre 40 y $80 \mathrm{kms}$ de ancho.

Diferentes consecuencias podría traer consigo un acto de este tipo, entre las más importantes encontramos:

- La interrupción de las líneas de comunicación y logísticas internas,

- La interrupción del flujo comercial desde y hacia diferentes zonas del país,

- La inauguración de un proceso de anexión territorial por una potencia vecinal.

- Golpe psicológico a los efectivos armados en conflicto y a la sociedad en su conjunto.

En el primer caso, la solución paliativa a esta posibilidad provendría de un sistema de defensa zonal, en donde se divide el país de acuerdo a las características geoestratégicas del territorio nacional. Este sistema operacional consistiría, someramente, en dotar de plena autonomía, táctica, estratégica y logística, a unidades militares completas. Es así como estas son compuestas por batallones de tanques, de infantería motorizada y mecanizada, de artille- 
ría, apoyadas, a su vez, por un soporte logístico en toda la fase, ya sea esta de penetración en territorio enemigo, como en la defensa pasiva de su propio territorio. En cuanto a este último punto, la defensa zonal permite una reducción de las líneas de comunicaciones interiores, tanto logísticas como operacionales. ${ }^{21}$

En cuanto al segundo punto, este se encuentra íntimamente asociado al fenómeno de ocupación expuesto anteriormente. La gran diferencia radica en que la ocupación de una franja transversál del territorio chileno -cortándolo en dos- es que en esta coyuntura la amputación territorial no es total, sino que parcial. En otras palabras, el segmento bajo ocupación se limita al territorio continental y no marítimo, lo que permitiría mantener, teóricamente, un contacto con la otra sección del territorio nacional por vía marítima, sólo en el caso, evidentemente, de mantener una supremacía aérea y naval.

Finalmente, la perpetuación de una división territorial podría, eventualmente, venir acompañada por un proceso de anexión.

Si hablamos de la percepción de amenaza chilena en términos de amenaza general y específica, una característica central cabe señalar: la amenaza gene$\mathrm{ral}$ ha coincidido en ocasiones con la potencial y la especifica con la real.

En efecto, tradicionalmente, Chile ha identificado a tres unidades estatales regionales como fuentes de amenaza directa para la seguridad nacional: Argentina, Perú y Bolivia.

Ahora bien, históricamente, es con estos tres países que Chile a mantenido vigente hipótesis de conflicto ${ }^{22}$ y es con estos tres países que el factor de amenaza ha evolucionado de la intención a la cuasi acción.

El periodo actual de acercamiento político entre las naciones del Cono Sur así como la voluntad declaratoria de integración regional ha puesto en desuso, oficialmente, las hipótesis de conflicto en vigor. ${ }^{23}$ No obstante este fenómeno, las fuentes de amenaza no han desaparecido, sino que éstas se convirtieron en fuentes de amenaza potenciales: existen, pero las condiciones no están dadas para que evolucionen a una de tipo real.

\footnotetext{
21 En este sentido, no es lo mismo desplazar suministros militares a lo largo de $1000 \mathrm{kms}$ desde los centros de aprovisionamiento hasta la zona de fricción bẻlica que hacerlo en un eje de $500150 \mathrm{kms}$. 22 En este caso, la llamada variante $\mathrm{H}_{3} \mathrm{~V}$ o hipótesis vecinal con tres enemigos atacando juntos surge como la hipótesis más extrema en un conflicto regional.

${ }^{23}$ En relación a este punto consultar, Leyton, S. Cristian., "De la Seguridad Cooperativa a la Seguridad por la Cooperación/Disuasión. Un Estudio de la Política de Seguridad por la Convergencia Estratégica", Revista Fuerzas Armadas y Sociedad, Fl.ACSO, Año 14, No.4, Octubre-Diciembre 1999.
} 
La historia reciente y pasada nos muestra que en el sistema internacional los acuerdos interestatales sólo dependen, en su acatamiento, de la voluntad política del los gobiernos y regímenes en oficio. Por esta razón toda política de conciliación debe ser alimentada por actos concretos de transparencia militar y de confianza mutua. Ahora bien, no obstante lo anterior, la existencia y el mantenimiento de FF.AA muy bien entrenadas, capacitadas y equipadas continua siendo el mejor garante de la integridad territorial y de la soberanía nacional, y ello, hasta que el factor de hostilidad interestatal disminuya, evolucionando la amenaza de una real a una potencial.

Ya hemos revisado los puntos de referencia geoestratégicos del territorio chileno así como la percepción de amenaza que éstos generan. Pasemos a continuación a abordar, las líneas directoras de lo que deberían ser las respuestas chilenas frente a esta realidad vecinal.

\section{Política de defensa chilena:}

\section{¿doctrinalmente defensiva pero operacionalmente ofensiva?}

Oficialmente, la política de defensa chilena, tal como nos lo es presentada en el Libro Blanco de la Defensa, es por esencia defensiva. ${ }^{24}$ No obstante esta política declaratoria, el Reglamento de la Conducción Estratégica del Ejército de Chile, la Doctrina Básica de la Fuerza Aérea y el libro Estrategia Naval del Vicealmirante H. Justiniano nos enseñan que los principios conductores de la estrategia operacional de estas tres ramas de las fuerzas armadas chilenas son esencialmente ofensivas. ${ }^{25}$ ¿Estamos hablando entonces de una política de defensa doctrinalmente defensiva pero operacionalmente ofensiva?

Los límites editoriales impuestos en esta ocasión no me permiten avanzar detalladamente en este cuestionamiento, sin embargo lo que sí es factible es extrapolar ideas básicas que nos permitan darnos una idea de lo que debería ser la política de defensa chilena, y ello, a la luz de la geoestrategia así como de la geopolítica del territorio chileno. ${ }^{26}$

\footnotetext{
24 "La Política de Defensa de Chile es, en el plano político-estratêgico, de carácter disuasivo y su orientación fundamental es defensiva". Libro de la Defensa Nacional de Chile, Ministerio de la Defensa Nacional de Chile, p. 88. 2s Tres son los principios que se repiten en cada uno de los reglamentos de conducción estratëgica: Ofen. siva, sorpresa y concentración de los medios.

${ }^{26}$ Cuando hablo de geoestrategia lo hago en función del control del espacio en têrminos de seguridad militar, mientras que cuando se hace referencia a la geopolítica del territorio nacional lo hago en función del territorio en términos de influencia política. Por un lado se trata de pensar el territorio o el espacio en términos de seguridad militar, por otro pensar el espacio en têrminos de capacidad de influencia política más allá de las fronteras nacionales.
} 


\section{¿Qué política de defensa?}

Dos grandes principios estratégicos tipifican, generalmente, las Políticas de Defensa de los Estados: las Políticas de Defensa pasivas y las activas. En otras palabras, aquellas que, dadas ciertas limitaciones materiales, optan por absorber territorialmente un ataque sorpresa y luego contraatacar, y aquellas que, dadas determinadas condiciones geoestratégicas, optan por anticipar el desencadenamiento de un ataque adversario enemigo con el fin de dictar los términos, el lugar y el momento de la conflagración.

Tomemos como ejemplo la evolución de la política de defensa de la OTAN en Europa durante la Guerra Fría. ${ }^{27}$

A comienzos de los años 70, la política de defensa de la OTAN podía ser calificada como pasiva. Esta consistía a reaccionar defensivamente frente a un ataque del Pacto de Varsovia a través de un repliegue táctico de sus fuerzas, esperando, con ello, la llegada de refuerzos desde los Estados Unidos y del Reino Unido, principalmente. Se trataba, en esencia, de sacrificar, transitoriamente, territorio por tiempo. Para ello, se eligió una defensa en profundi$d a d$, vale decir, desplazar sucesivamente las líneas defensivas hacia el interior del territorio de la Alemania Occidental . ${ }^{28}$ El objetivo último era, pues, retrasar el avance de las fuerzas de elite soviéticas, o los Grupos de Maniobra Operacional (GMO) y del Pacto, en su ingreso al territorio de la Alianza. Dados los impresionantes dispositivos defensivo aéreos soviéticos así como su concentración en una extensión limitada de territorio, las fuerzas de la OTAN veían limitadas la posibilidad de operacionalizar una estrategia de ataque en profundidad, vale decir, más allá de la línea de frente o FEBA (Fonvard Edge of the Battle Area) sólo con la ayuda de sistemas de aviones de ataque estratégico.

Ahora bien, la problemática ligada a la adopción de dicha estrategia de defensa pasiva era lo inaceptable de su puesta en operación: 30\% de la población alemana occidental así como $25 \%$ de su base industrial se concentraba en una extensión de sólo $100 \mathrm{kms}$, la que corría en forma paralela a lo largo

\footnotetext{
7 Ver, Jacquelin, C. Davis y Robert, L. Pfootzgraf., "The Atlantic Alliance and US Global Strategy ", Special Report, Institut for Foreign Policy Analysis, Cambridge, septiembre 1983, pagina $29 \cdot 41$. Ver además, Boyd, D. Sutton (ed), "Deep Attack Concept and the Defence of Central Europe ", Survival, Vol. XXVI, Número 2, marzo-abril 1984, paginas 50-70.

${ }^{28}$ La extensión transitoriamente cedida era de aproximadamente $40 \mathrm{kms}$ al interior del territorio alemán occidental.
} 
de la frontera con su similar Oriental. Los daños colaterales y directos que una defensa en profundidad implicaba eran prohibitivos para la existencia misma del Estado de Alemania Occidental.

A mediados de los años 70, esta doctrina cambia ligeramente. Se adopta una estrategia de defensa avanzada (Forward Defense). Básicamente, se trataba de neutralizar casi in sitiu, vale decir en la frontera misma, los asaltos militares del Pacto. ${ }^{29}$ Por ello, era una doctrina de negación de los objetivos político-militares soviéticos. La problemática de esta estrategia era su carácter reactivo, vale decir, que sólo sería puesta en práctica una vez el ataque sorpresa soviético consumado y no en el momento o antes que este fuese activado. Esta característica reactiva de la estrategia de Forward Defense implicaba, entonces, daños colaterales a las zonas frontaleras alemanas occidentales así como el permitir a las fuerzas del Pacto dictar los términos de la fricción bélica.

Finalmente, en noviembre 1984 el SACEUR (Suprem Allied Commander of Europe), aprueba y decide implementar la doctrina Follow-on-ForcesAttack (FOFA). ${ }^{30} \mathrm{La}$ doctrina Rogers (Nombre del General Bernard Rogers instigador de la nueva política militar), consistía atacar en profundidad las fuerzas del segundo escalón soviéticas -la reserva estratégica- con el fin de impedir que las unidades GMO fuesen apoyadas en su fase de penetración en territorio occidental. ${ }^{3 l}$ Esta estrategia era, en gran parte, de atrición por cuanto se daba como objetivos militares, no sólo las fuerzas de reserva del segundo escalón del Pacto, sino que también, sus principales puntos de aprovisionamiento, rutas, y aeropuertos.

En resumen, esta estrategia toma en consideración la geoestrategia del territorio de la Alemania Occidental al buscar la profundidad estratégica faltante en el territorio enemigo y no en el suyo. De esta forma, la política de defensa de la OTAN en Europa evoluciona de una pasiva, esperando, en un primer momento absorber el primer golpe del Pacto (Defensa en profundidad),

29 En este caso, la distancia entre la frontera internacional de ambas Alemanias y las líneas del frente de fricción militar era de aproximadamente $3 \mathrm{kms}$. En resumen, la defensa hacia delante estaba limitada a menos de 5 kilómetros de las concentraciones demográficas e industriales de la Alemania Occidental.

${ }^{30}$ La doctrina FOFA de la OTAN podia ser operacionalizada conjuntamente con la doctrina del US Army conocida como Airland Battle, la cual era eminentemente anti-fuerza y limitada a una extensión promedio de $150 \mathrm{kms}$ de distancia del punto cero del frente de batalla.

${ }^{3 *}$ La penetración en territorio del Pacto era de 300 a $600 \mathrm{kms}$ en su máxima extensión.

${ }^{32}$ Ahora bien, no obstante el hecho que las doctrinas de la OTAN constituyen, ante todo, doctrinas de interdicción, es decir, de ataque de la retaguardia con el fin de impedir la llegada de refuerzos militares, esta también forma parte de la política de defensa activa, pero en su lectura de estrategia anti-logistica. 
luego contenerlo (Defensa hacia adelante), para finalmente optar por una política de defensa activa-reactiva, de ataque en la profundidad (Deep Attack) del territorio enemigo. ${ }^{32}$ No obstante esto último, no se trataba de embestir preventivamente las fuerzas del Pacto, sino que de esperar el primer asalto soviético y de desgastar las fuerzas destinadas a colmar las aperturas hechas por los Grupos de Maniobra Operacional de la exURSS. Si las fuerzas de defensa antiaéreas soviéticas impedían la plena puesta en práctica de esta doctrina a mediados de los años 70, el desarrollo y adquisición por parte de las fuerzas occidentales de mísiles inteligentes (GPM), por ejemplo, más precisos y capaces de atravesar mayores distancias, por ende, con una alta capacidad de supervivencia, abrieron la puerta a su adopción final.

A la luz de los argumentos precedentes, ¿cómo debería operacionalizarse la política de defensa chilena? La similitud geoestratégica entre ambos casos, el chileno y el alemán es evidente. La realidad geoestratégica y geopolítica nacional impondría una política de defensa doctrinalmente defensiva pero operacionalmente ofensiva. En otras palabras, Chile expande y transforma el principio de política de defensa defensiva acordándola a su vulnerabilidad aérea y terrestre. Chile no tiene ambiciones territoriales, pero dada la geoestrategia de sus centros vitales operacionalizará una estrategia de reacción militar preventiva.

De esta forma, la configuración territorial chilena, su emplazamiento y características geográficas asociadas al factor de hostilidad vecinal, así como al emplazamiento de sus zonas vitales deberían, en teoría, imponer al Estado chileno la puesta en obra de una Política de Defensa activa, vale decir, de ataque anticipado, transfiriendo las hostilidades, lo más pronto posible, en territorio enemigo, y ello, en caso de sentir amenazada la santuarización de sus zonas vitales. Chile no podría negociar espacio por tiempo ya que carece de este último factor defensivo.

Los círculos decisorios político-militares chilenos al optar por una política de defensa activa buscarían aniquilar, por el uso de la sorpresa y del ataque indirecto, cualquier concentración de fuerzas potencialmente hostiles desplegadas en la proximidad de la frontera. En este sentido, Chile puede optar por una política de defensa doctrinalmente defensiva -Chile no hará uso de la fuerza en primer lugar-, pero operacionalmente ofensiva- Chile usará la fuerza en caso de sentirse amenazado. La problemática política impuesta por esta estrategia se resuelve por medio de la adopción de una política declaratoria 
de casus bellis, vale decir, explicitando toda acción y capacidad vecinal pudiendo poner en peligro la seguridad territorial y soberana de la nación y por lo tanto, justificando una acción de fuerza defensiva, incluso antes de ver operacionalizada la amenaza potencial. Entre los casus bellis más susceptibles de ser declarados encontramos, una concentración de fuerzas inhabituales en la zona fronteriza, la adquisición de ciertos tipos de armas a carácter ofensivos (desarrollo o adquisición de una capacidad balística), la creación de alianzas militares, el bloqueo de ciertos pasos marítimos así como el cierre del espacio aéreo internacional a vuelos nacionales, entre otros.

La cuestión central es saber si las condiciones materiales, políticas y coyunturales están dadas para la adopción y puesta en práctica de tal doctrina militar. Parece evidente, a la luz de las nuevas relaciones interestatales a nivel vecinal, marcadas por la cohabitación entre la disuasión y la cooperación, que tales condiciones han dejado de materializarse. De esta forma, mientras el factor fuerza aun genera percepciones de amenaza reales, el factor de cooperación limita su ventilación a un nivel eminentemente disuasivo permitiendo avanzar por el difícil pero necesario camino hacia la conciliación de intereses nacionales.

A título de conclusión y con el fin de extender el debate, ¿en qué medida, entonces, la geoestrategia y geopolítica del territorio nacional determinan realmente la Política de Defensa chilena, sobre todo en este período de convergencia estratégica? ¿En qué medida, otras variables, internas y externas, influencian o deberían influenciar la política de defensa y de seguridad de Chile, no obstante el imperativo impuesto por su geografía, por los factores de amenaza tradicional así como por su geoestrategia nacional? 